\title{
Electron Microscope Observation of the Austenite and the Martensite in High Aluminium Steel (Supplement)*
}

\author{
By Tsugio Tadaki** and Ken'ichi Shimizu**
}

\begin{abstract}
A supplementary examination of the quenched structure of an $\mathrm{Fe}-9.70 \mathrm{wt} \% \mathrm{Al}-1.46 \mathrm{wt} \% \mathrm{C}$ alloy has been carried out by means of transmission electron microscopy and diffraction, in order to clarify whether the retained austenite is ordered in the $\mathrm{Cu}_{3} \mathrm{Au}$ type, as reported in the previous paper (Trans. JIM, 12 (1971), 386), or it is composed of the $\kappa$ carbide and the disordered $\gamma$ austenite, as suggested by Oshima and Wayman (Met. Trans., 3 (1972), 2163). It is again verified that the retained austenite is basically ordered in the $\mathrm{Cu}_{3} \mathrm{Au}$ type, and a peculiar shape, which has markedly been observed in fundamental spots from the retained austenite, is shown to be due to an accompaniment of diffuse satellites with the austenite reflection spots. The appearance of satellites suggests the existence of such a modulation as that carbon atoms are concentrated in distorted regions near the second type antiphase domain boundaries. Some of the concentrated regions with carbon atoms possibly form the perovskite-type $\kappa$ carbide, as suggested, since superlattice reflections from the retained austenite exhibit two kinds of intensities. The $c / a$ ratio of the tetragonal martensite in this alloy is determined to be 1.14 from a definite twinned diffraction pattern.
\end{abstract}

(Received April 1, 1974)

\section{Introduction}

In a previous electron microscope study(1) of the martensitic transformation in an $\mathrm{Fe}-9.70$ $\mathrm{wt} \% \mathrm{Al}-1.46 \mathrm{wt} \% \mathrm{C}$ alloy, the following results were obtained: (1) Electron diffraction patterns taken from the retained austenite exhibited extra reflection spots which could be explained as superlattice reflections from the $\mathrm{Cu}_{3} \mathrm{Au}$ type ordered structure. Each of the reflection spots was accompanied by a short cross streak. (2) Dark field electron micrographs formed by using a 100 superlattice reflection from the ordered austenite revealed bright and dark domain contrasts with a rectangular shape, the size of the domains being about $120 \AA$ on the average. The rectangular contrasts were attributed to the formation of antiphase domains in the retained austenite. From these results, the present authors concluded that the $\gamma$ phase at high temperature underwent an ordering reaction prior to martensitic transformation

* This paper was originally read at the Annual Fall Meeting of Japan Inst. of Metals, Hakata, October, 1973.

** The Institute of Scientific and Industrial Research, Osaka University, Yamadakami, Suita, Osaka 565, Japan.

Trans. JIM upon quenching, and that the ordered $\gamma$ phase contained antiphase domain boundaries of the second type.

Very recently, however, Oshima and Wayman $^{(2)}$ gave another explanation for the appearance of extra reflections and domain contrasts. According to them, the extra reflections originate from the perovskite-type $\kappa$ carbide $\left(\mathrm{Fe}_{3} \mathrm{AlC}\right)$ epitaxially precipitated in disordered $\gamma$ austenite, and the bright and dark contrasts in dark field electron micrographs correspond to the $\kappa$ carbides and disordered $\gamma$ austenite, respectively. Thus they concluded that the retained austenite was not ordered but composed of the $\kappa$ carbide and disordered $\gamma$ austenite, in contrast with our previously drawn conclusion. Their conclusion was based on the following results: (1) Fundamental reflection spots from the retained austenite exhibited a peculiar shape, which was considered to be due to a composition of two reflection spots closely spaced. (2) Dark field electron micrographs formed by such a composite fundamental reflection showed moiré-like images. (3) Extra superlattice reflection spots were not exactly located at the middle points between the composite fundamental reflection spots. (4) The superlattice reflections with $h+k+l=$ even were weaker than those with 
$h+k+l=$ odd, as expected from the perovskitetype $\kappa$ carbide. If all of these results were true, their conclusion would be valid since lattice parameters of the $\kappa$ carbide and $\gamma$ austenite are very close to each other ${ }^{(1)(3)(4)}$. However, taking into account of the composition of the present steel, such a conclusion seems to be unreasonable because the bright domains account for the greater part of the specimen volume while the dark domains explain a little remainder.

At all events, the respective conclusions in the above two experiments should more clearly be verified. In order to do so, it is necessary to examine in detail the $\kappa$ carbide itself and the origin of the peculiar shape of fundamental reflection spots. For this purpose, the present supplementary experiment has been carried out on the quenched structure of high aluminium steel, and could verify definitely that the retained austenite was basically ordered in the $\mathrm{Cu}_{3} \mathrm{Au}$ type. The results obtained will be reported below.

\section{Experimental Procedure}

The steel used is the same as that in the previous experiment, the composition being $\mathrm{Fe}-9.70 \mathrm{wt} \% \mathrm{Al}-1.46 \mathrm{wt} \% \mathrm{C}$. The method of specimen preparation has been described elsewhere $^{(1)}$. In the present study, in order to examine the $\kappa$ carbide itself and some of its connections with the martensitic transformation in the steel, the following heat treatments are newly adopted according to the phase diagram after Löhberg and Schmit ${ }^{(5)}$. That is, disk shaped specimens are sealed in evacuated quartz capsules, austenitized at $1200^{\circ} \mathrm{C}$ for $6 \mathrm{hr}$, held for $1 \mathrm{hr}$ at $1100^{\circ} \mathrm{C}$ where both the $\kappa$ and $\gamma$ phases are stable, and then rapidly quenched into water at $0^{\circ} \mathrm{C}$.

The specimens were electropolished in the same way as before ${ }^{(1)}$ and examined by means of electron microscopy and diffraction. The electron microscope, Hitachi HU-11D, operating at $100 \mathrm{kV}$ was used.

\section{Results and Discussion}

Photograph 1 is an example of the electron

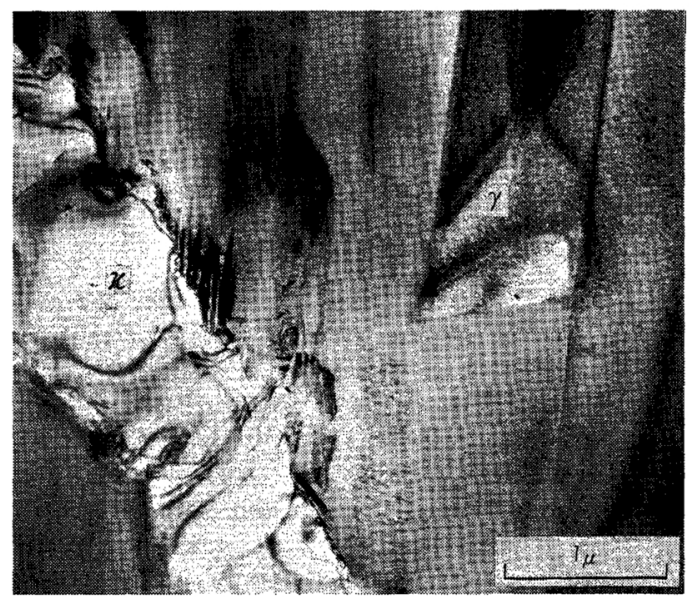

Photo. 1 Electron micrograph of $\mathrm{Fe}-9.70 \mathrm{wt} \% \mathrm{Al}-$ $1.46 \mathrm{wt} \% \mathrm{C}$ steel as-quenched from $1100^{\circ} \mathrm{C}$, showing the coexistence of $\kappa, \gamma$ and $\alpha^{\prime}$ crystals.

micrographs taken from the specimens heattreated as above. There are seen one $\kappa$ carbide and retained $\gamma$ austenite as well as $\alpha^{\prime}$ martensites, as expected beforehand and also verified by electron diffraction. The $\kappa$ carbide has an indeterminate shape and seems to have no connection with the surrounding martensites. Furthermore, the carbide has no internal structure revealing a smooth contrast, while the retained austenite as well as the martensites have some characteristic fine structures. These facts suggest that the carbide has no relation with the $\gamma \rightarrow \alpha^{\prime}$ martensitic transformation in this steel.

The structurelessness of the $\kappa$ carbide and the fine structure of the retained austenite can more clearly be known from Photos. 2 and 3, respectively. Photographs 2(a) and (b) are magnified bright and dark field electron micrographs, respectively, taken from within another $\kappa$ carbide, and the (b) is of the same area as the (a) and was taken by using an 100 reflection. Both the bright and dark field micrographs show no fine structure in the carbide, and all reflection spots of the corresponding diffraction pattern (c), which is in the (001) orientation, are very sharp in accordance with the structurelessness. The sharpness of reflection spots enables us to determine the lattice parameter of the $\kappa$ carbide. Analyzing many diffraction patterns exhibiting sharp spots, the lattice parameter was 

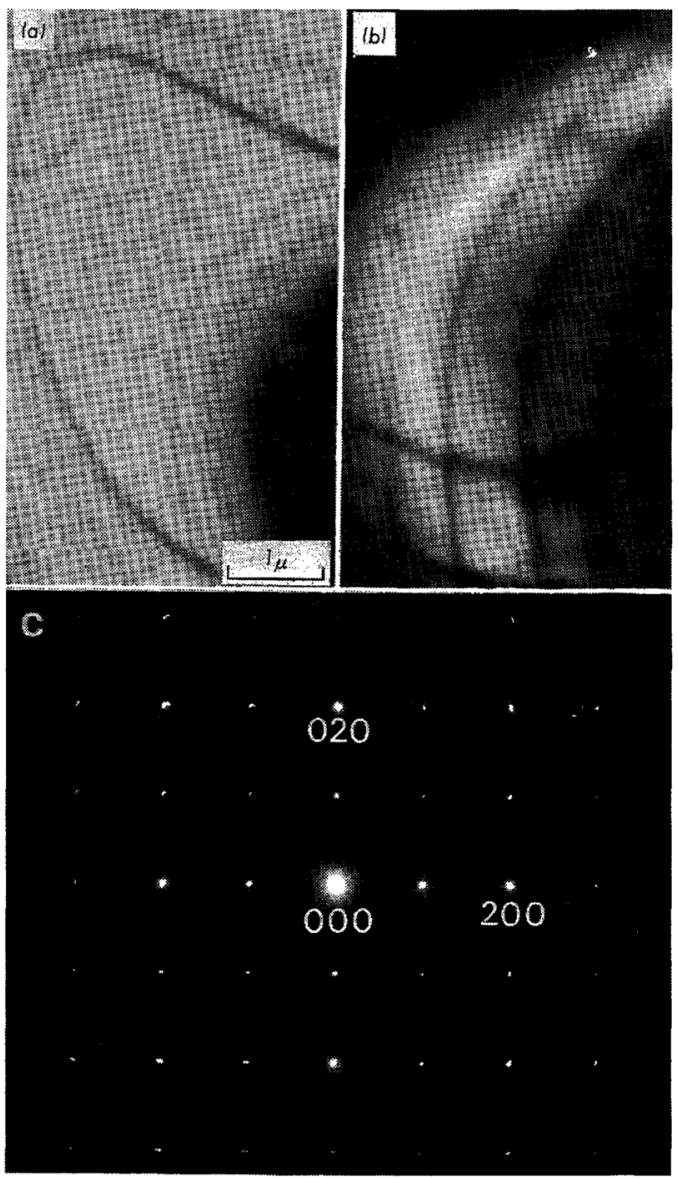

Photo. 2 (a) Electron micrograph of a $\kappa$ carbide with the (001) orientation. (b) Dark field image of (a) formed by a 100 reflection. (c) Electron diffraction pattern taken from the whole area of (a), showing the (001) zone's of $\kappa$.

known to be consistent with the values (3.74 3.78 $\AA$ ) reported so $\operatorname{far}^{(4)}$.

On the other hand, Photos. 3(a) and (b), which are bright and dark field electron micrographs, respectively, of the retained austenite, clearly show some fine structures. The (b) is of the same area as the (a), and is formed by using the 100 superlattice reflection of (c), which is the corresponding diffraction pattern taken from the whole area of (a) and is in nearly the (001) orientation. The fine structure in (b) can be characterized by bright and dark rectangular domains, as pointed out in the previous experiment. All superlattice reflections in (c) seem to have nearly the same intensity, taking into account of a deviation of the electron beam
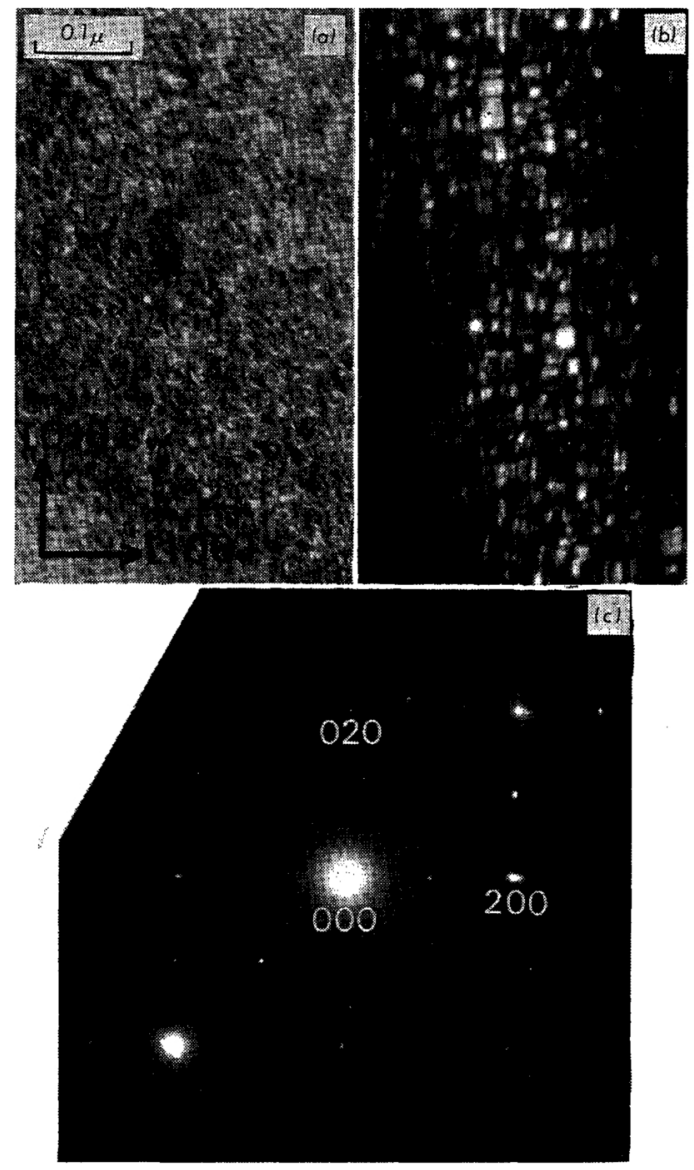

Photo. 3 (a) Electron micrograph of a retained $\gamma$ crystal with nearly (001) orientation. (b) Dark field image of (a) formed by a 100 extra reflection. (c) Electron diffraction pattern taken from the whole area of (a).

from the (001) orientation. Thus, the present authors concluded before that the austenite matrix was ordered in the $\mathrm{Cu}_{3} \mathrm{Au}$ type, and interpreted that the bright and dark domains corresponded to antiphase domains with the second type boundaries. On the other hand, Oshima and Wayman interpreted the bright and dark domains as the $\kappa$ carbides and disordered $\gamma$ austenite, respectively. To examine which interpretation is correct is the main subject of the present experiment.

Photographs 4(a) and (b) show the [001] and [110] zone patterns, respectively, of the retained austenite exhibiting the characteristic fine structure as mentioned above. In both (a) and (b), extra reflection spots in question are seen as 


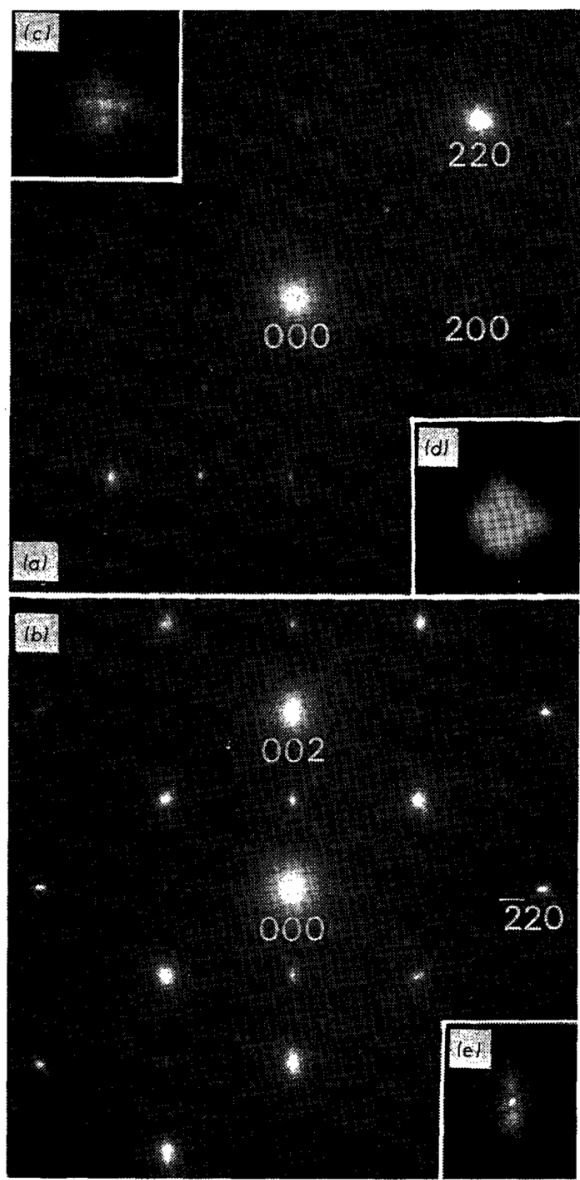

Photo. 4 Typical electron diffraction patterns taken from retained $\gamma$. (a) and (b) show the [001] and the [110] zonal patterns, respectively. (c), (d) and (e) show fine structures of the 220 and the 002 reflections, respectively, appearing with various exposure times.

well as fundamental reflection spots, and all the reflection spots are peculiarly elongated along the $\langle 001\rangle$ directions. In order to examine the details of the reflection spots, successive diffraction patterns were taken by changing only the exposure time. An example with respect to the 220 reflection is shown in (a), (c) and (d), and another example with respect to the 002 reflection is shown in (b) and (e). The 220 reflection spot, which appears rather round in (a), becomes as if it were a doublet splitting along the radial direction when it was taken with an exposure time a little shorter than in (a), as seen in (d). However, that the doublet is not an essential shape is obvious from (c) which was taken with an exposure time much shorter than in (a). That is, the (c) reveals that the 220 reflection is actually accompanied with very diffuse but clearly distinguishable four satellites. The 002 reflection spot, which appears elongated along the [001] direction in (b), comes out in (e) to be accompanied with two diffuse satellites when it was taken with an exposure time a little shorter than in (b). Thus, a peculiar shape observed as to fundamental reflection spots can be attributed to diffuse satellites around the main reflection spots. The three-dimensional shape of the satellites may be like a football lengthened along a $\langle 001\rangle$ direction.

As mentioned above, Oshima and Wayman attributed the peculiar shape of fundamental reflections to two reflections from the $\kappa$ carbide and disordered $\gamma$ austenite. As an instance of the experimental evidence, they showed a 220 reflection under nearly the same situation as in Photo. 4(d), and interpreted it in such a way that the inner and outer spots corresponded to those from the $\kappa$ carbide and disordered $\gamma$ austenite, respectively. However, the above experiment clearly showed that the 220 reflection was accompanied with four satellites, and then that their interpretation was not reasonable.

Next, in order to know the exact locations of extra reflection spots in question, interdistances among reflection spots were measured by tracing diffraction patterns with a microphotometer. Photograph 5 is an example of the microphotometer curves, which was traced between the 000 and 002 reflections in Photo. 4(b). There can be found the 001 extra reflection exactly at the middle point between the 000 and 002 main reflections, as indicated. By using such 001 and 110 superlattice reflections as well as other fundamental main reflections, the lattice parameter of the retained austenite phase was determined to be $3.67 \AA$. This value is different from that of the $\kappa$ carbide $(3.74 \sim 3.78$ $\AA$ ), and is consistent with that previously determined as well as that reported by Watanabe and Wayman $^{(4)}$ for the austenite matrix. These results clearly indicate that the extra reflections are of the retained austenite (not the $\kappa$ carbide), and therefore that the retained austenite is 


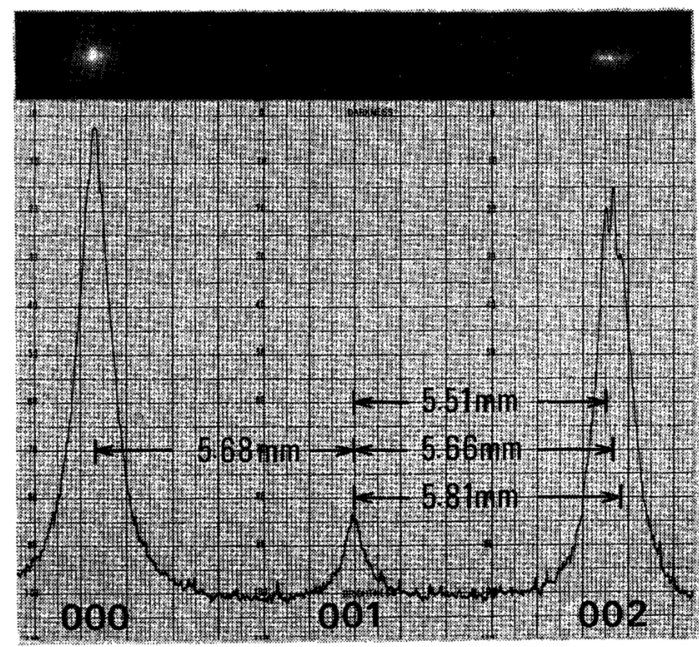

Photo. 5 Microphotometer trace between 000 and 002 reflections in Photo. 4(b).

basically ordered in the $\mathrm{Cu}_{3} \mathrm{Au}$ type, contrary to the Oshima and Wayman's suggestion. The ordering reaction is supposed to take place during the quenching as in the cases of $\beta$ phase in noble metal alloys. Therefore, the ordered domains may not be able to be so large, the size being about $130 \AA$. Such a size of domains is not energetically unfavorable since much smaller domains have been observed in other alloy systems.

Now, the above microphotometer tracing also provides some useful information about the appearance of satellites around the main austenite reflections. If such satellites are due to a composition and/or spacing modulation in the solid solution as generally considered, the separations between the main and satellites reflections suggest that the wave length of the modulation should be about $130 \AA$. Futhermore, satellites of the low angle side are always stronger in intensity than those of the high angle side, as clearly seen in Photo. 5, indicating that both the composition and spacing modulations are caused and that they are in phase to each other. Taking into account of that the wavelength of the modulation is nearly the same as the average size of rectangular antiphase domains observed in dark field electron micrographs, Photo. 3(b) in both the present and previous $^{(1)}$ papers, and also that many of domain boundaries are of the second type, the modulation may be such that carbon atoms are concentrated in the distorted regions near the second type antiphase domain boundaries. Some of the concentrated regions with carbon atoms possibly form the perovskite-type $\kappa$ carbides as suggested by Oshima and Wayman, since superlattice reflections with $h+k+l=$ even are somewhat weaker than those with $h+k+l=$ odd. The steel used in the Oshima and Wayman's work contained lower aluminium and higher carbon contents than those in the present work. The lower aluminium content might hinder a tendency to the ordering, and the higher carbon content might promote a
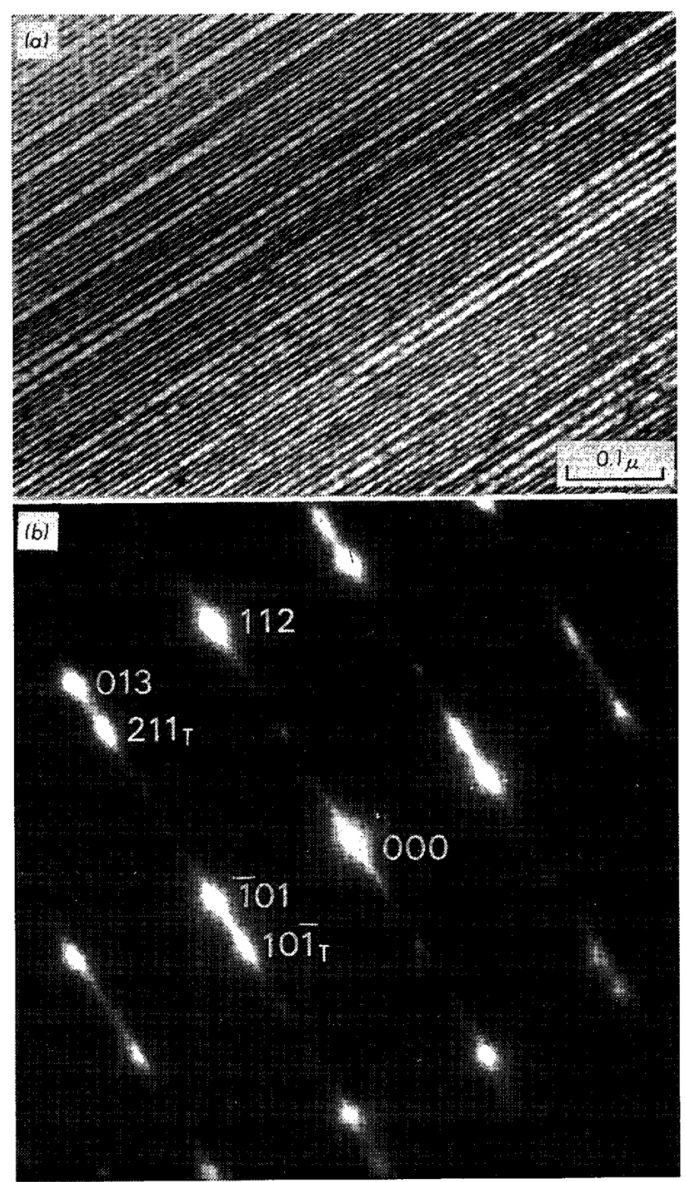

Photo. 6 (a) Electron micrograph of internal twin faults in an $\alpha^{\prime}$ crystal, the (112) fault plane being perpendicular to the foil surface. (b) Electron diffraction pattern taken from the whole area of (a), the electron beam being parallel to the $[1 \overline{3} 1]$ and the $[\overline{1} 3 \overline{1}]_{T}$ directions of the martensite matrix and of its twin, respectively. 
tendency to the $\kappa$ carbide formation. In such a way, some differences might be caused between their work and ours.

Finally, a brief description of the martensites produced in the quenched steel will be given. Photograph 6(a) is an electron micrograph showing internal defects of a martensite. Although their defects are known to be twins ${ }^{(6)}$, definite twinned diffraction patterns have not yet been obtained so far. In the present experiment, a definite twinned diffraction pattern is obtained as shown in Photo. 6(b), and it undoubtfully indicates that the internal defects are the (112) twins. Now, this twinned diffraction pattern is slightly different from that in $b c c$ martensites. That is, in $b c c$ martensites, twin reflection spots are located at $1 / 3$ positions along the 112 reciprocal lattice vector. However, the present twin reflection spots can be found at positions shifted from the $1 / 3$ positions. Since the amount of the shift depends on the magnitude of tetragonality, precise measurements of the shift should bring about the magnitude of tetragonality. In this way, the axial ratio $c / a$ is determined to be 1.14. This value is well consist- ent with those reported in the references ${ }^{(4)(7)}$.

\section{Acknowledgments}

The authors are grateful to Emeritus Prof. Z. Nishiyama for his critical reading of the manuscript and usefull comments, and also to Prof. C. M. Wayman and Dr. Oshima for their valuable comments. This work was partly supported by a Grant-in Aid for Fundamental Scientific Research (Sōgo, 1973) from the Ministry of Education.

\section{REFERENCES}

(1) T. Tadaki, K. Shimizu and T. Watanabe: Trans. JIM, 12 (1971), 386.

(2) R. Oshima and C. M. Wayman: Met. Trans., 3 (1972), 2163.

(3) M. Watanabe and C. M. Wayman: Met. Trans., 2 (1971), 2229.

(4) L. S. Palatnik, I. A. Tananko and Yu G. Bobro: Sov. Phys. Cryst., 9 (1964), 163.

(5) K. Löhberg and W. Schmidt: Arch. Eisenhuttenw., 12 (1937), 607.

(6) Z. Nishiyama, K. Shimizu and M. Harada: Trans. JIM, 11 (1970), 152.

(7) Z. Nishiyama and M. Doi: J. Japan Inst. Metals, 8 (1944), 305. 